\title{
Accurate Pedestrian Indoor Navigation by Tightly Coupling Foot-Mounted IMU and RFID Measurements
}

\author{
Antonio Ramón Jiménez Ruiz, Fernando Seco Granja, José Carlos Prieto Honorato, and Jorge I. Guevara Rosas
}

\begin{abstract}
We present a new method to accurately locate persons indoors by fusing inertial navigation system (INS) techniques with active RFID technology. A foot-mounted inertial measuring units (IMUs)-based position estimation method, is aided by the received signal strengths (RSSs) obtained from several active RFID tags placed at known locations in a building. In contrast to other authors that integrate IMUs and RSS with a loose Kalman filter (KF)-based coupling (by using the residuals of inertial- and RSS-calculated positions), we present a tight KF-based INS/RFID integration, using the residuals between the INS-predicted readerto-tag ranges and the ranges derived from a generic RSS path-loss model. Our approach also includes other drift reduction methods such as zero velocity updates (ZUPTs) at foot stance detections, zero angular-rate updates (ZARUs) when the user is motionless, and heading corrections using magnetometers. A complementary extended Kalman filter (EKF), throughout its 15-element error state vector, compensates the position, velocity and attitude errors of the INS solution, as well as IMU biases. This methodology is valid for any kind of motion (forward, lateral or backward walk, at different speeds), and does not require an offline calibration for the user gait. The integrated INS + RFID methodology eliminates the typical drift of IMU-alone solutions (approximately $1 \%$ of the total traveled distance), resulting in typical positioning errors along the walking path (no matter its length) of approximately $1.5 \mathrm{~m}$.
\end{abstract}

Index Terms-Dead reckoning, inertial navigation, Kalman filters, position measurement, RFID tags.

\section{INTRODUCTION}

A $S$ GPS is essential for outdoor navigation, there is also a growing need for accurate and continuous indoor localization systems. Consequently, this topic has received significant scientific research attention during the last years. There are several location-aware application fields that can benefit from indoor localization, such as, intelligent spaces, personal or asset tracking, guidance of persons with mobility problems, or firstresponders.

Two main research approaches are used in the indoor positioning problem: 1) solutions that rely on the existence of a network of receivers or emitters placed at known locations (beacon-based solutions); and 2) solutions that mainly rely on dead-reckoning methods with sensors installed on the person or object to locate (beacon-free solutions). Beacon-based approaches, estimate position by trilateration or triangulation from a set of measured ranges or angles, respectively. They are usually termed local positioning systems (LPSs), in analogy to GPS, although they normally use a different technology, such as ultrasound, short-range radio (WiFi, UWB, RFID, Zigbee, etc.) or vision. Some LPS surveys can be found in [1], [2].

The second approach (beacon-free or dead-reckoning) is preferable in some applications since it does not depend on a preinstalled infrastructure. During the last decade several beacon-free methods based on inertial measuring units (IMUs) have been proposed for the localization of persons [3]-[5]. These methodologies, often called pedestrian dead-reckoning (PDR) solutions, can integrate the user step lengths and heading angles at each detected step, to estimate the user's position [6]-[9]; or, alternatively, integrate accelerometer and gyroscope readings of a foot-attached IMU (by strapdown inertial navigation system (INS) mechanization [10]) to compute the position and attitude of the person [11]-[14].

IMU-based PDR solutions have the inconvenient of accumulating errors that grow with the path length. This drift problem is common to every dead-reckoning position estimation method, although it can be minimized by using highperformance IMUs. However, for a portable self-contained PDR solution there is a limit to the weight, volume and power consumption, and consequently low-performance microelectromechanical systems (MEMS) IMUs have to be used [15]. Unfortunately, MEMS acelerometers and gyroscopes are subject to significant random noise and bias [16] that have to be estimated on-line in to partially attenuate their drift effects. A more effective way to eliminate the accumulated error, is to fuse PDR with some indoor absolute positioning references, such as a received signal strength (RSS)-based local positioning system or RSS-LPS [17] or time-of-arrival (TOA) positioning systems (TOA-LPS) [18]. This PDR+LPS integrated concept, as already implemented in many outdoor applications fusing INS and GPS [19], has the potential to provide an accurate driftfree positioning solution.

RFID technology has several practical advantages over other LPS approaches: it has an easier deployment (just stick several RFID tags in the environment); no synchronization cables are required (as opposite to TOA methods such as UWB); andthe 
density of nodes can be adapted and changed according to the requirements of the application (i.e., a higher flexibility than in other preinstalled networks such as the existing Access Point in WiFi networks). For these reasons, we will use RFID technology in this paper, and the RSS obtained from several active RFID tags, placed at known locations in a building, will be processed to cancel out the existing PDR drift. However, the use of RSS is challenging due to the fading and the stochastic behavior of Signal Strength values (caused by indoor obstacles, reflections, body absorption, and so on).

The integration of RSS information with inertial data has been proposed recently by a some authors; some of them use the RSS from access points (AP) in a building ([20]-[22]), and others use the RSS from RFID tags ([23]-[26]). Nevertheless, no matter if the RSS is obtained from AP or RFID tags, the algorithms to process RSS can be the same. Two main approaches to integrate RSS measurements with Inertial data can be found in the literature: 1) RSS-centric; and 2) Inertial-centric. The RSS-centric approach is focused in position estimation from the RSS data (by fingerprinting, Bayesian estimation or fitting data to a path-loss model); some corrections from the inertial sensor (in the form of incremental stride distances traveled) are fed into the estimation filter to improve the movement model and to smooth the estimated trajectory (otherwise quite noisy paths are normally obtained using only RSS values [17], [27], [28]). For example, in [21] a WiFi RSS-based fingerprinting method is presented to estimate the position using a particle filter implementation; the stride length (SL) estimation is fed (only distance, no orientation) in the particle filter as a movement model to sample the new particles positions in a circumference having a normally distributed radius with mean equal to the measured SL. A similar range-based movement model is found in [22] where step lengths are used to adapt the transition probabilities in a Hidden Markov Model (HMM).

In the Inertial-centric integration methods, the main focus of the estimation is on the inertial processing (INS or SL-based) and the position information estimated with any RSS-LPS method is used to update the position state of the IMU-based PDR estimation. In [23] a SL-based PDR algorithm, that uses information from several IMUs in the body, is updated in position and orientation each time a user crosses a door. An RFID tag is placed in the top center of the door's frame; when the RFID is detected (close-range coverage) the PDR position is reset with the corresponding door's position (prestored in a database), and the yaw orientation is also reseted to the door's orientation. A similar close-range concept is used as well in [29], in this case with a method denominated "RFID time-based CoO". Another SL-based PDR method implemented with a particle filter (and map information) is improved by weighting the particles by a probabilistic $\mathrm{P}$ (RSS|Position) measurement model [20]. In [24], [25], [30] a INS-based PDR method is updated with position and velocity estimations from a parallel RFID probabilistic fingerprinting positioning method. A separate Kalman filter $(\mathrm{KF})$ is used for the INS estimation, which is fed with the residuals between inertial- and RFIDcalculated positions.

The RSS + IMU integration methods presented until now in the literature for PDR, in both RSS-centric and Inertial-centric approaches, rely on the estimation of the position from RSS information as an independent or separate building block, i.e., they perform a so called loose integration with IMU signals. This loose approach is know in the GPS/INS community to be less effective than other approaches that integrate raw sensor data at a lower processing level (called a tight integration) [31]. Some of the benefits of tight integrations are: Drift reduction even with only one node (RSS value) available; Feedback of sensor error parameters (IMU bias, model constants), among others. In [26] a path-loss model relating distance to RSS is presented; the authors suggest that it could be possible to integrate the RSS data with the IMU using a tight KF-based estimation, however they do not implement or detail how it could be implemented, and consequently no localization tests are presented.

In this paper, we present, implement and test a new KF-based INS/RFID tight integration method using the residual between the INS-predicted range to tag, and the range derived from a generic RSS path-loss model. Our approach also includes zero velocity updates (ZUPTs) at detected foot stances, zero angularrate updates (ZARUs) at still phases, and heading drift reduction using magnetometers. A complementary extended Kalman filter (EKF), throughout a 15-element error state vector [12], [14], compensates position, velocity and attitude errors of the INS solution, as well as IMU biases. Our methodology is valid for any kind of motion (forward, lateral or backward walk), and does not require a specific off-line calibration, neither for the user gait, nor for the location-dependent RSS fading in the building. In contrast to other works, several quite long trajectories (more than 500-m long each) are evaluated in different areas of an indoor building. The results demonstrate the effectiveness of the methodology in eliminating positioning drifts.

The paper is organized as follows. The next section presents the IMU and RFID sensors used for the indoor location tests. Section III describes the KF-based INS/RFID tight integration method, and finally, Section IV performs an evaluation of several indoor localization tests. Conclusions and future research directions are given in last section.

\section{SENSORS AND INFRASTRUCTURE USED}

For estimating the user's position and displacement, we use two sensors: one IMU to provide the inertial data, and a set of several RFID tags with one long-range RFID reader, to get the RSS information.

\section{A. $I M U$}

We use a commercially available IMU, model MTi from Xsens Technologies B.V. (Enschede, The Netherlands; www.xsens.com). Its size is $58 \times 58 \times 22 \mathrm{~mm}$ (length $\times$ width $\times$ height), and it weighs $50 \mathrm{~g}$. It is configured to provide inertial data at $100 \mathrm{~Hz}$.

The IMU has three orthogonally oriented accelerometers, three gyroscopes and three magnetometers. The accelerometers and gyroscopes are solid state MEMS with capacitative readout, providing linear acceleration and rate of turn, respectively. Magnetometers use a thin-film magnetoresistive principle to measure the earth 's magnetic field. The performance of individual MEMS sensors within the MTi IMU are summarized in 
TABLE I

PERFormanCE OF INDIVIDUAL SENSORS IN XSENS IMU

\begin{tabular}{l|l|l|l}
\hline & accelerometers & gyroscopes & magnetometers \\
\hline Axes & 3 & 3 & 3 \\
Full Scale (FS) & $\pm 50 \mathrm{~m} / \mathrm{s}^{2}$ & $\pm 300 \mathrm{deg} / \mathrm{s}$ & $\pm 750 \mathrm{mGauss}$ \\
Linearity & $0.2 \%$ of FS & $0.1 \%$ of FS & $0.2 \%$ of FS \\
Bias stability & $0.02 \mathrm{~m} / \mathrm{s}^{2}$ & $1 \mathrm{deg} / \mathrm{s}$ & $0.1 \mathrm{mGauss}$ \\
Bandwidth & $30 \mathrm{~Hz}$ & $40 \mathrm{~Hz}$ & $10 \mathrm{~Hz}$ \\
Max update rate & $512 \mathrm{~Hz}$ & $512 \mathrm{~Hz}$ & $512 \mathrm{~Hz}$ \\
\hline
\end{tabular}

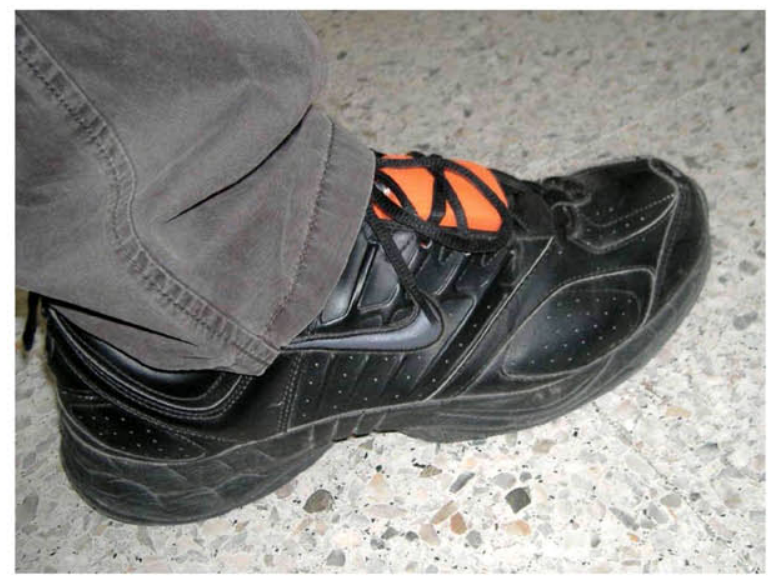

Fig. 1. Xsens IMU attached to the right foot using the shoe's laces.

Table I (Xsens specifications). As expected, they suffer from a significant bias that also varies over time (bias stability).

This work uses the IMU mounted on the foot of a person to take advantage of ZUPTs at foot stances. Fig. 1 shows the Xsens sensor fixed to the right foot of a person, using the shoe's laces. The exact position and orientation of the IMU on the foot is not important for the algorithms that we use to process the sensor data (INS-based PDR [9]).

\section{B. RFID}

We use active RFID technology from the company RF Code Inc. (Austin, TX, USA; www.rfcode.com). In our solution we use several tags located at fixed positions within a building, and a portable RFID reader (Fig. 2).

1) RFID Tags: We use active tags model M100, that are battery-powered RF transmitters operating in the $433 \mathrm{MHz}$ radio band. Every tag broadcasts its unique ID and a status message at a periodic rate $(1 \mathrm{~Hz})$ programmed at the factory. The size of each tag is $46.74 \times 34.28 \times 11.68 \mathrm{~mm}(\mathrm{~L} \times \mathrm{W} \times \mathrm{H})$. Each tag weights $14.1 \mathrm{~g}$, including a replaceable coin-cell lithium battery (model CR2032). The expected lifetime is more than seven years with one emission every 12.5 seconds (according to the manufacturer), so for $1 \mathrm{~Hz}$ emission rate the battery lifetime it is expected to be about 6 months. Tags can be put into sleep mode (emissions disabled) using a tag activity controller, model A600 from RFCode; this option is useful to preserve batteries when the system is not to be used during long periods of time.

2) RFID Reader: Among all the available RF Code readers, we use the model M220 because it is a light-weight portable battery-powered reader. It processes the signals coming from neighboring active RFID tags, and can communicate to a

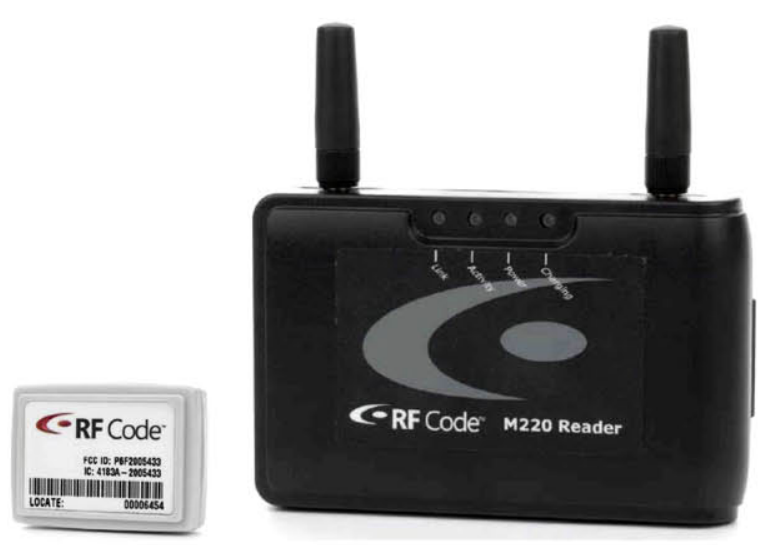

Fig. 2. RFID equipment from RFCode Inc. Left: RFID active tag model M100. Right: RFID reader model M220.

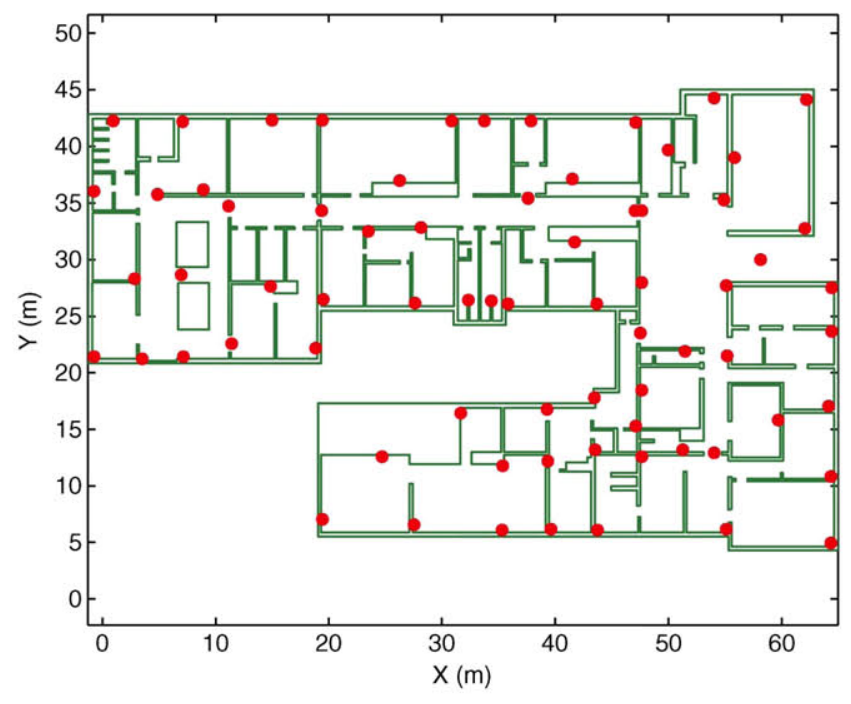

Fig. 3. Distribution of 71 RFID tags (red circles) in our main building.

Bluetooth-enabled host processor (PC, PDA, smart phone), and also by a wired USB-serial connection (Bluetooth 1.1 and USB 2.0). The maximum read-out distance between the reader and the tags is up to $70 \mathrm{~m}$ in ideal conditions (free space). The RFID reader is equipped with two short range stub antennas. It is also possible to install 1/4-wave articulated helical antennas for operating at larger distances. Each reader reports the RSS information at each antenna for every in-range tag. The reader size is $111 \times 76.5 \times 25.1 \mathrm{~mm}(1 \times \mathrm{w} \times \mathrm{h})$, and it only weights $147 \mathrm{~g}$.

\section{Deployment of RFID Tags}

We have installed 71 RFID tags in our CAR-CSIC main building $\left(2200 \mathrm{~m}^{2}\right)$, a brick made construction with a majority of wooden doors. These tags are stuck on the walls at approximately $2-\mathrm{m}$ height (above most furniture or windows). The tag distribution (Fig. 3) is somehow random, attempting to obtain an homogeneous density, but trying to get at least three non-aligned close-range tags from any indoor location (good dilution-of-precision, or DOP [31]). Some tags are at some strategic doorways that we believed could ease the location during transitions from one corridor to a room, or viceversa. 


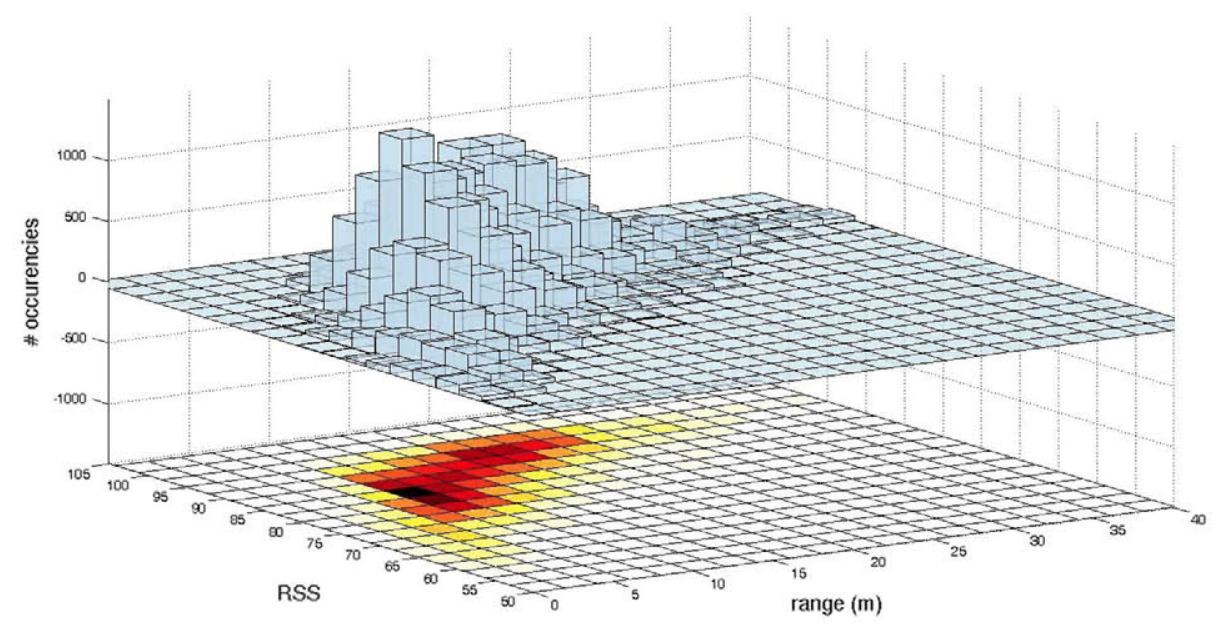

Fig. 4. Histogram of RSS values versus the tag-to-reader distance.

The tag density in the distribution of Fig. 3 is about 1 tag every $30 \mathrm{~m}^{2}$. It is relatively high, but we decided to use enough tags in to be able to study the influence of the tag density on the final positioning results. In this way, it is straightforward to simulate a lower tag density by simply not using some of the RSS data coming from some selected tags. An optimum tag density, as a trade-off between deployment cost and positioning accuracy, will be studied in Section IV-D.

\section{RSS Data Acquisition}

Before defining in Section III the methodology to integrate RFID measurements in the positioning method, we first present some RSS data-collection experiments obtained by placing the RFID reader at several static positions within the building. The collected RSS data plotted versus the tag-to-reader range (see Fig. 4) clearly shows the typical stochastic nature of RSS measurements in complex indoor environments caused by multiple fading, reflections, refractions, and multi-paths. This raw data representation also gives insight on how RSS values depends on the tag-to-reader distance. A generic model representing the dependence of signal strength with range will be defined in Section III and used in the integrated IMU+RFID positioning algorithms.

The provided RSS value of RFCode reader is in fact a value that ranges between 40 and 110 , where a value of 40 corresponds to the maximum signal strength, and values approaching 110 are the weakest signals.

The data presented in Fig. 4 corresponds to a total of 32 different reader positions along the main hall and corridor of the CAR-CSIC building. In each position, measurements were taken for one minute, with four different orientations (approximately 15 seconds for each orientation). So the total acquisition time was 32 minutes, and the resulting number of RSS measurements was 46.687 , that represents $34 \%$ of the total number of emissions in 32 minutes $(136.320$ emissions $=$ 32 minutes $\times 60$ (emissions/minute $) \cdot \operatorname{tag} \times 71$ tags $)$. The fact that only $34 \%$ of the total emissions were detected is caused by a decreasing probability of detection at larger ranges (see Fig. 5). Note that only a low percentage of detections are produced above $25 \mathrm{~m}$.

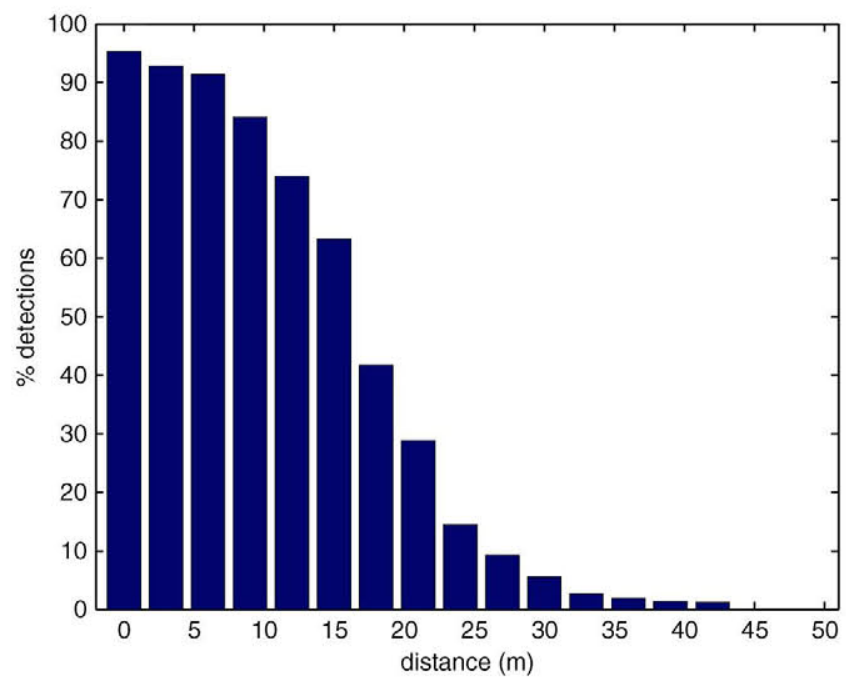

Fig. 5. Probability of tag detection clearly depends on the tag-to-reader distance.

\section{INTEGRATEd IMU+RFID POSITIONING METHOD}

Before presenting the RFID integration method, we quickly review in the next subsection the basic framework for the IMU-alone estimation (the core of RFID integration).

\section{A. IMU-Alone IEZ+ Method}

The IMU-alone IEZ + method is a PDR positioning method, that was recently presented by Jiménez et al. [14]. This method is an extension of the ZUPTs Kalman-based methodology presented by Foxlin [12]. The name of IEZ+ method is a contraction of these acronyms: IMU-EKF-ZUPT-Extended, that stands for "Inertial Measurement Unit-Extended Kalman Filter-Zero Velocity Update-Extended". The reader of this paper is referred to Jiménez et al. [14] for implementation details, nevertheless some key aspects will be highlighted here.

IEZ+ performs an INS mechanization on the foot's position based on IMU readings. This INS process, which is prone to accumulate errors due to IMU biases, is corrected by the 15 -element state vector of an EKF: $\mathbf{X}=\left[\delta \mathrm{At}, \delta \omega^{b}, \delta \mathrm{Po}\right.$, $\left.\delta \mathrm{Ve}, \delta \mathrm{a}^{b}\right]$. This vector contains the estimated biases for the 


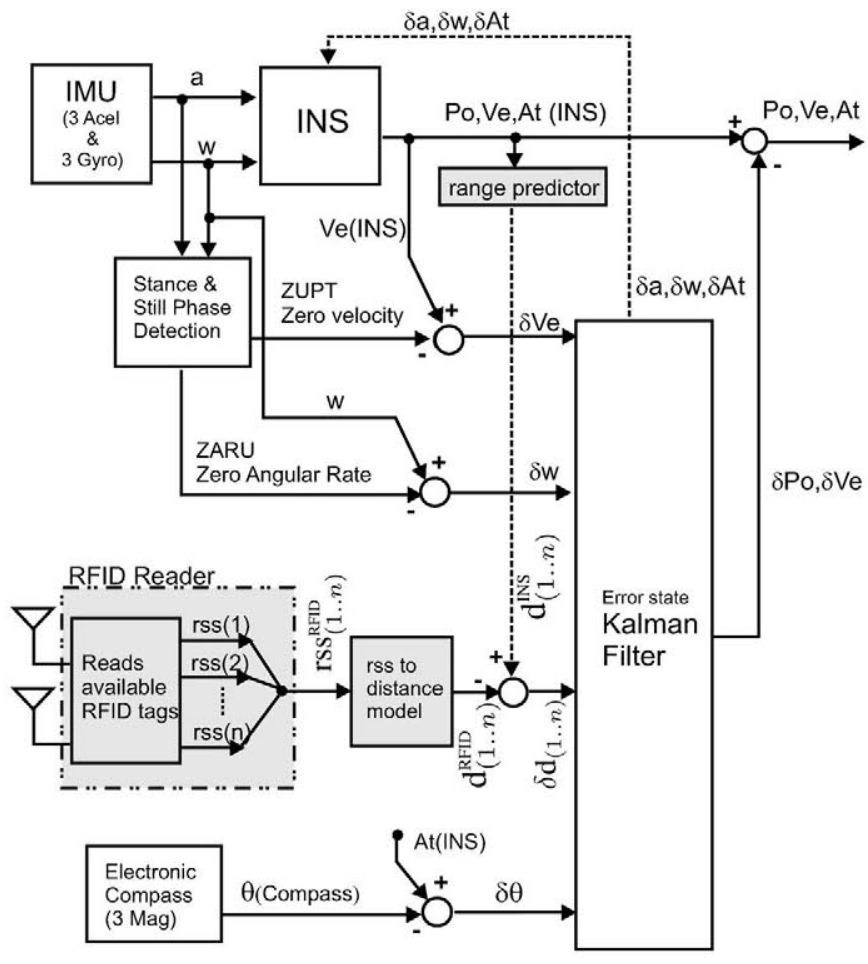

Fig. 6. IEZ + methodology [14], integrated with RFID measurements for drift-free pedestrian position estimation. Additional blocks for RFID integration are highlighted in gray color.

accelerometers and gyroscopes $\left(\delta \mathrm{a}^{b}\right.$ and $\delta \omega^{b}$, respectively), as well as the $3 \mathrm{D}$ errors in orientation or attitude $(\delta \mathrm{At})$, position $(\delta \mathrm{Po})$, and velocity $(\delta \mathrm{Ve})$. Fig. 6 represents a block diagram of the IEZ + methodology (ignore the light-gray blocks that are the additional components of the integrated RFID solution).

The EKF filter is executed at a $100-\mathrm{Hz}$ refresh rate. Most of the time only predictions are computed, but updates with measurements (corrections) are also performed at the same rate during foot stances. Hence, the EKF is updated with velocity measurements by the ZUPTs strategy each time the foot is on the floor; also with the angular rate of gyroscopes when the foot is totally stationary (no walking, e.g., standing or sitting). The latter update process is called ZARU, and provides a very good method (fully observable) to quickly find an approximation of gyroscope biases. IEZ+ also performs heading updates using the magnetometer sensor to limit the drift.

The IEZ + method, using only the self-contained information of the IMU, has proven to be a very reliable PDR method, with accumulated errors of approximately $1 \%$ of the total traveled distance [12], [14]. However, over long-distance trajectories this dead-reckoning method cannot avoid to progressively diverge from the true path. The integration of external RFID measurements within the IEZ + (next section) will help to solve this problem.

\section{B. Integration of RFID Measurements}

We aid the IEZ+ method by means of a tight integration using range residuals estimated from RFID signal strengths. In contrast to other approaches ([20]-[24], [26]), which initially estimate the position using a separate RFID-LPS system and then incorporate positions into the $\mathrm{KF}$ in a loose integration, we use the range-based tight approach which is known to have better performance in GPS and other range-based application systems [31].

The implemented RFID-aided INS indoor pedestrian navigator is depicted in Fig. 6. The whole block diagram corresponds to the IEZ+ methodology that has been complemented or expanded (to include the RFID information) with three additional blocks: 1) RFID reader; 2) Range predictor; and 3) RSS-todistance model (blocks in light-gray in Fig. 6).

The first extra block in Fig. 6 ( $R F I D$ reader) represents the used RFID reader, and its outputs: several RSS values acquired from different in-range tags. This RFID data is continuously recorded in a buffer by the reader as received from the tags. We collect the available RSS information in the reader at a $10 \mathrm{~Hz}$ update rate. Since the reader has two antennas, most in-range tags are detected by both antennas simultaneously, so we obtain two RSS values from each tag. In our implementation we use the mean of these two RSS values per each detected tag. If $n$ tags are detected in a $0.1 \mathrm{~s}$ time interval, then $n$ RSS mean values (denoted as $\operatorname{RSS}_{(1 . . n)}^{\mathrm{RFID}}$ ) are used as measurements.

The second additional block in Fig. 6 is a range predictor, which gives the distance between the current position estimated by the INS mechanization, and the known position of tag $i$. Each individual predicted tag-to-reader range, $d_{i}^{\text {INS }}$, is computed as

$$
d_{i}^{\mathrm{INS}}=\sqrt{\left(X^{\mathrm{INS}}-X_{i}\right)^{2}+\left(Y^{\mathrm{INS}}-Y_{i}\right)^{2}}
$$

where $X_{i}$ and $Y_{i}$ are the known 2D -position coordinates of $\operatorname{tag} i$ (prestored in a database); and $X^{\mathrm{INS}}$ and $Y^{\mathrm{INS}}$ are the current position coordinates estimated by the IEZ + architecture at the INS output $\left(\mathrm{Po}=\left\{X^{\mathrm{INS}}, Y^{\mathrm{INS}}, Z^{\mathrm{INS}}\right\}\right)$. Usually, more than one tag is detected $(n)$, in that case, the complete set of range predictions to the $n$ tags is denoted by $d_{(1 . . n)}^{\mathrm{INS}}=\left\{d_{i}^{\mathrm{INS}} \mid i=\right.$ $(1 . . n)\}$.

The third additional block in Fig. 6 (RSS-to-distance model) transforms the RSS values into range data. Given the mean RSS value from tag $i$, this model estimate the distance, $d_{i}^{\text {RFID }}$, between tag $i$ and the RFID reader, based only on RSS values, i.e., $d_{i}^{\mathrm{RFID}}=f\left(\mathrm{RSS}_{i}\right)$. The range residual, $\delta d_{i}$, between the tagto-reader predicted distance, $d_{i}^{\text {INS }}$, and the distance estimated with the model, $d_{i}^{\mathrm{RFID}}$, is obtained as

$$
\delta d_{i}=d_{i}^{\mathrm{INS}}-d_{i}^{\mathrm{RFID}} .
$$

For a set of $n$ detected tags we used the notation $\delta d_{(1 . . n)}$ for the residuals. All available range residuals, as indicated in Fig. 6, are fed into the EKF, at a $10 \mathrm{~Hz}$ rate. The success of this RFID integration depends on the correctness of the model relating the RSS with the true tag-to-reader distance. The model used to relate RSS to range is presented in detail in the next subsection.

\section{RSS to Distance Model}

The RSS value registered in a reader from the emission of a tag, depends on their separation, or tag-to-reader range, but also on some other unpredictable stochastic factors (specially 
indoors). The attenuation caused by the distance $d$ between an emitter and a reader, is known as the path loss [32]. This attenuation is inversely proportional to the distance between emitter and receiver raised to the so called path loss exponent $p$. This exponent $p$ equals two for an ideal spherical dispersion in free space, is lower than two for propagation along waveguides (e.g., corridors), and larger than two when multipath, refraction, or shadowing occurs in the propagation media (typical in buildings). The received power $\left(P_{R}\right)$ at the reader can be modeled as

$$
P_{R} \propto P_{T} \cdot \frac{G_{t} \cdot G_{r}}{4 \pi d^{p}}
$$

where $P_{T}$ is the transmitted power at the emitter, $G_{t}$ and $G_{r}$ are the antenna gains of transmitter and receiver, respectively, $d$ is the distance between emitter and receiver, and $p$ is the path loss exponent. Using logarithmic units in (3), and considering that RSS is the received power in decibels, we obtain

$$
\mathrm{RSS}=\mathrm{RSS}_{0}-10 \cdot p \cdot \log _{10}\left(\frac{d}{d_{0}}\right)+v
$$

where $\mathrm{RSS}_{0}$ is a mean RSS value obtained at a reference distance $d_{0}$, and $v$ is a Gaussian random variable with zero mean and standard deviation $\sigma_{\mathrm{RSS}}$ that accounts for the random effect of shadowing [28]. From (4), the maximum likelihood estimate of distance $d$ is given by

$$
d=d_{0} \cdot 10^{\frac{\mathrm{RSS}_{0}-\mathrm{RSS}}{10 \cdot \mathrm{p}}} .
$$

We obtained the unknown parameters $\mathrm{RSS}_{0}$ and $p$, by fitting the experimental data presented in Fig. 4 to the RSS-distance model (5). We found, for a reference distance $d_{0}$ of $1 \mathrm{~m}$, that RSS $_{0}$ equals 60 and $p$ is -2.3 (both parameters have been rounded). The minus sign in the path loss exponent accounts for the inverse dependence of RFCode RSS read-out versus power (decreasing values of RSS represent stronger signals).

The experimental standard deviation of RSS values, $\sigma_{\mathrm{RSS}}$, has been found to be almost independent of distance $\left(\sigma_{\mathrm{RSS}}=6\right)$. To obtain the standard deviation of the estimated distance, $\sigma_{d}$, which is needed by the Kalman filter as an indication of the belief we have on the modeled range value, we use the following heuristic assumption: $\sigma_{d}$ must be equal to $\sigma_{\mathrm{RSS}}$ when the slope in model of (5) is one, and in general it should be reasonably estimated as inversely proportional to the slope of the distance model. If we differentiate (4) with respect to distance $d$ to obtain the slope, we get

$$
\frac{\partial \mathrm{RSS}}{\partial d}=-10 \cdot p \cdot \frac{1}{\ln (10)} \cdot \frac{d_{0}}{d} \cdot \frac{1}{d_{0}} .
$$

Consequently, the sought standard deviation of distance $\left(\sigma_{d}\right)$ to be used in our model is

$$
\sigma_{d}=\sigma_{\mathrm{RSS}} \cdot \frac{\ln (10) \cdot d}{-10 \cdot p} .
$$

This sigma model is linearly proportional to distance, so it gives low standard deviation values at short ranges (low uncertainty) and a larger sigma at long ranges (high uncertainty).

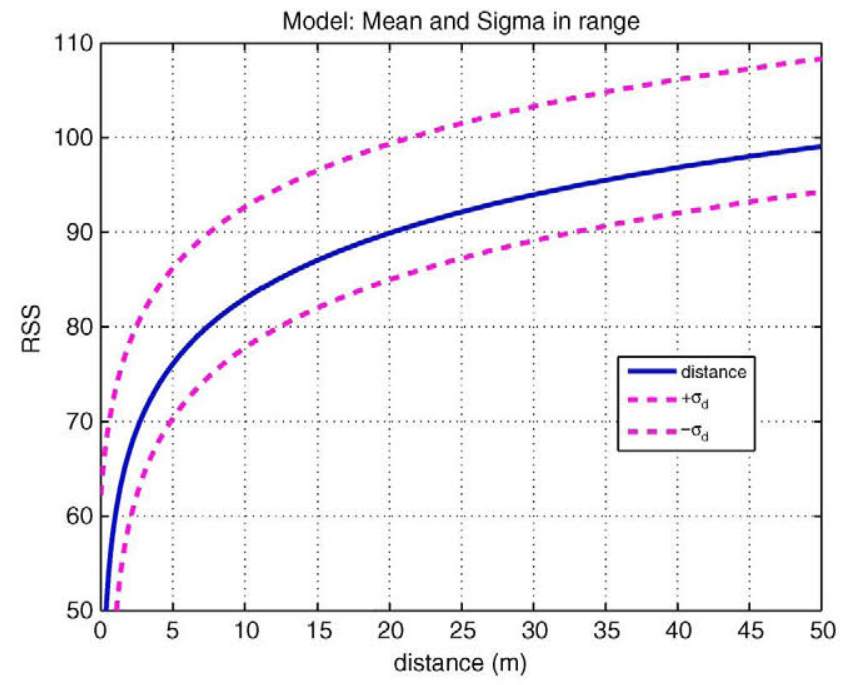

Fig. 7. RSS versus distance model. The solid line corresponds to the distance model (5) evaluated for several RSS values; the dashed line corresponds to the addition of $\pm \sigma_{d}$ (from (7)) to the distance model for every RSS value evaluated.

The combined model representation of RSS versus distance $\left(d\right.$ and $\left.\sigma_{d}\right)$ is depicted in Fig. 7. If for example, we have an RSS value of 90, then, using (5) (solid blue line in Fig. 7), we obtain an estimated range of $20 \mathrm{~m}$; the uncertainty of this range value is obtained with (7) (dashed lines), which gives a standard deviation in distance, $\sigma_{d}$ of about $12.5 \mathrm{~m}$ (symmetric at both sides of the solid line plot).

\section{Algorithm Implementation}

To give enough details of the implemented software, we present in Algorithm1's box the pseudocode of the main program, and that of the KF-based INS + RFID integration. Within this code, several function calls are not detailed since its functionality is clearly deduced from the function's name and the input and output parameters. The symbol of percentage, "\%," is used to mark the beginning of comments (also in blue color). Further INS and EKF implementation details were already presented in [14].

Algorithm 1 Integrated IMU + RFID Positioning Algorithm. More Kalman-related implementation details are in [14].

1: procedure MAIN \% Main program

2: $\quad\left[\right.$ State, Cov, Po, Ve, At, $\left.\mathrm{Po}_{\text {tags }}\right] \leftarrow$ Initialize();

3: STARTIMU\&RFIDMEASUREMENTS();

4: $\quad$ loop $\% 100 \mathrm{~Hz}$ rate

5: WaitNeXtIMUSAmPle; \% Max. $10 \mathrm{~ms}$

6: $\quad$ Data $_{\text {IMU }} \leftarrow\left[\boldsymbol{\omega}^{b}, \mathbf{a}^{b}, \mathbf{m}^{b}\right] \leftarrow$ GetiMU Data ();

7: $\quad[$ Stance, Still $] \leftarrow$ StepDetection Data $_{\text {Imu }}$;

8: $\quad$ if Stance then \% Foot at stance

9: $\quad \delta \mathrm{Ve} \leftarrow \mathrm{Ve}_{\text {INS }} \%$ ZUPTs

10: $\quad \theta_{\text {Compass }} \leftarrow$ Compass $\left(\right.$ Data $\left._{\text {IMU }}\right)$;

11: $\quad \delta \theta \leftarrow \theta_{\text {INS }}-\theta_{\text {Compass }} \%$ Compass

12: $\quad$ end if

13: $\quad$ if Still then \% No walk

14: $\quad \delta w \leftarrow w_{\text {IMU }} \%$ ZARU 


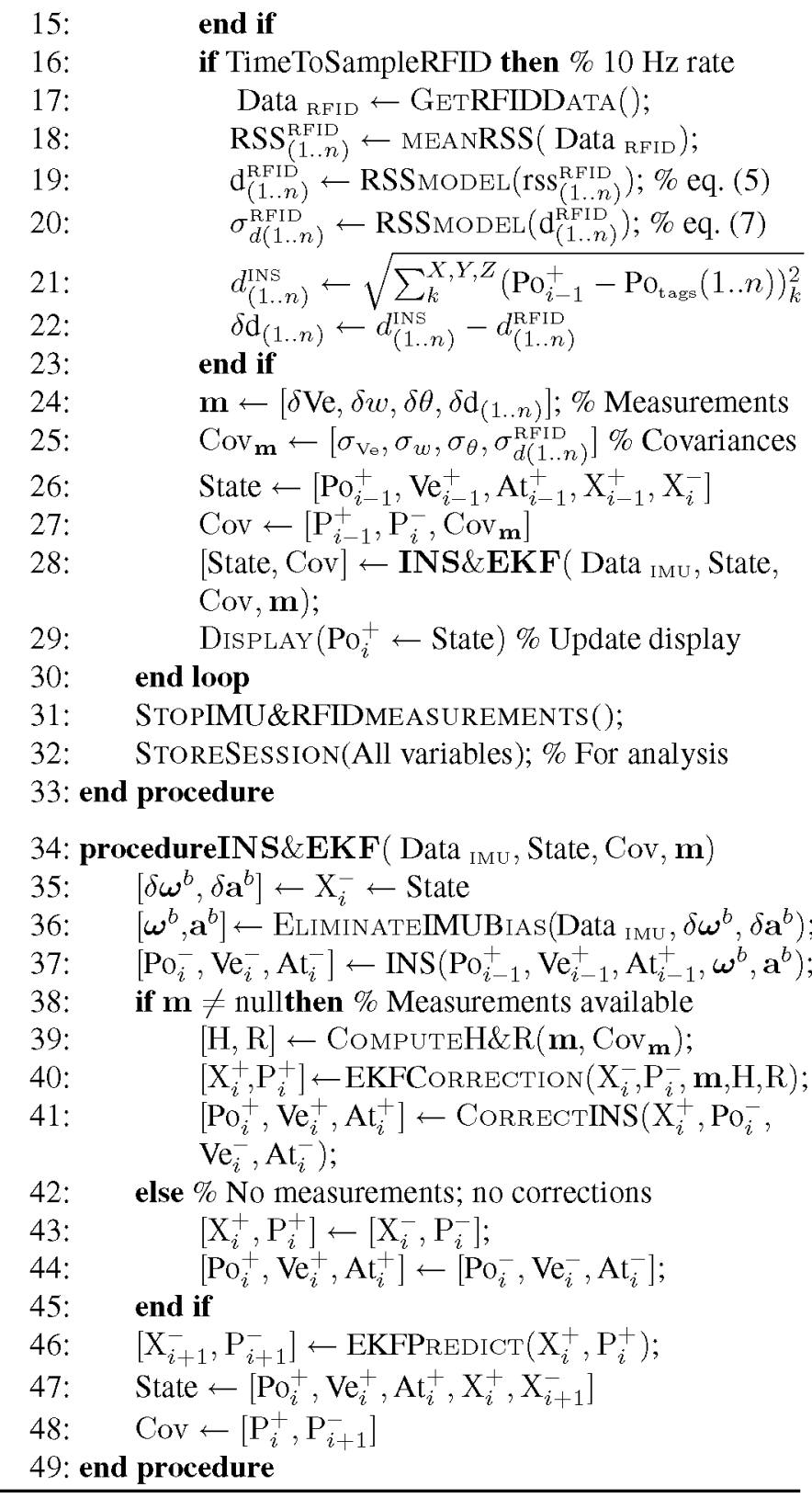

Our software methodology has been implemented in the Matlab programming language. It operates in real-time on a netbook computer. When a person using the navigation equipment walks inside or around the building, the estimated positions are displayed on-line over a building layout map on the computer screen ( $2 \mathrm{~Hz}$ position refresh rate with the netbook computer). Sensor data and estimated parameters are stored at the end of each test for subsequent analysis.

\section{INDOOR LOCALIZATION TESTS}

\section{A. IMU-Alone Estimation}

In this subsection, we analyze the performance of the proposed methodology without using the RFID information, i.e., the performance of the IEZ + method. The IEZ + method, already presented and assessed in Jimenez et al. paper [14], demostrated a positioning error of about $1 \%$ of the total travelled distance (TTD). The results were very satisfactory, but in that work the paths under evaluation consisted in just one repetition of a given trajectory.

Now, we present a set of four indoor tests in our main building, with different closed paths that were repeated several times until the accumulated positioning error was significant (larger than $5 \mathrm{~m}$ ). Although, we just use an IEZ+ processing (IMU-alone solution), during these tests we also recorded the sensed RFID data so as to have the opportunity to replicate exactly the same tests in next subsection, but in that case integrating both IMU and RFID data.

The registration of data was performed by a person wearing an IMU on the right foot and an RFID reader on the right side of his waist with both sensors connected by USB to a netbook computer. The person walked at a normal pace, approximately at $1 \mathrm{~m} / \mathrm{s}$ in the forward direction, along corridors and entering into some room labs. The doors in the building were opened to facilitate the navigation, but the system also works well if the person has to stop to open a door or to wait for another person in his path to pass. Some trajectories include $180^{\circ}$ turns at dead-ends.

The four tests together with the estimated trajectories using IEZ+ (IMU-alone) are displayed in Fig. 8. The start position is marked with a black square, and the final position with a black circle and a magenta arrow indicating the direction of the person. The small dots along the trajectory (approximately 1.4 $\mathrm{m}$ apart from each other along the path) represent the detected right-foot stances. The exact trajectory of the multiple loops in each path is not known with high accuracy (there is no groundtruth data), however we known with centimeter accuracy that the stop and start positions are the same. Therefore, the accumulated positioning error (used to evaluate the performance of positioning algorithms) is computed as the 2-D Euclidean distance between the start and stop positions.

As expected, the repetition of the same trajectory several times has finally caused a drift of the position estimation toward an arbitrary direction, and the total error grows proportionally to the path length or the number of iterations. In Fig. 8(a), for a 600 -m-long path obtained with 8 repetitions, the error is accumulated at a rate of almost $0.63 \mathrm{~m}$ per cycle, and the start/ stop total error is about $5 \mathrm{~m}$, i.e., $0.8 \%$ of total travelled distance. In Fig. 8(b), a 550-m-long path obtained with 13 repetitions, the error is accumulated at a rate of almost $0.77 \mathrm{~m}$ per cycle, and the final error is about $10 \mathrm{~m}$, i.e., $1.8 \%$ of total travelled distance. In Fig. 8(c), a 520-m-long path obtained with 8 repetitions, the error is accumulated at a rate of almost $0.7 \mathrm{~m}$ per cycle and the final error is $7.7 \mathrm{~m}$, i.e., $1.4 \%$ of total travelled distance. Finally, in Fig. 8(d), a 1000-m-long path is obtained repeating 8 times a cycle that includes indoor navigation, and a partial outdoor path along a patio. The error is accumulated at a rate of almost $1.4 \mathrm{~m}$ per cycle, and the total accumulated error is $11.5 \mathrm{~m}$, i.e., $1.1 \%$ of total travelled distance. In these 4 tests the averaged error percentage with respect to the TTD is $1.27 \%$.

\section{B. Integrated IMU+RFID Estimation}

It is expected that the results obtained in Fig. 8 should be corrected by aiding the IMU-alone processing with an absolute positioning reference given by the RFID localization system. 

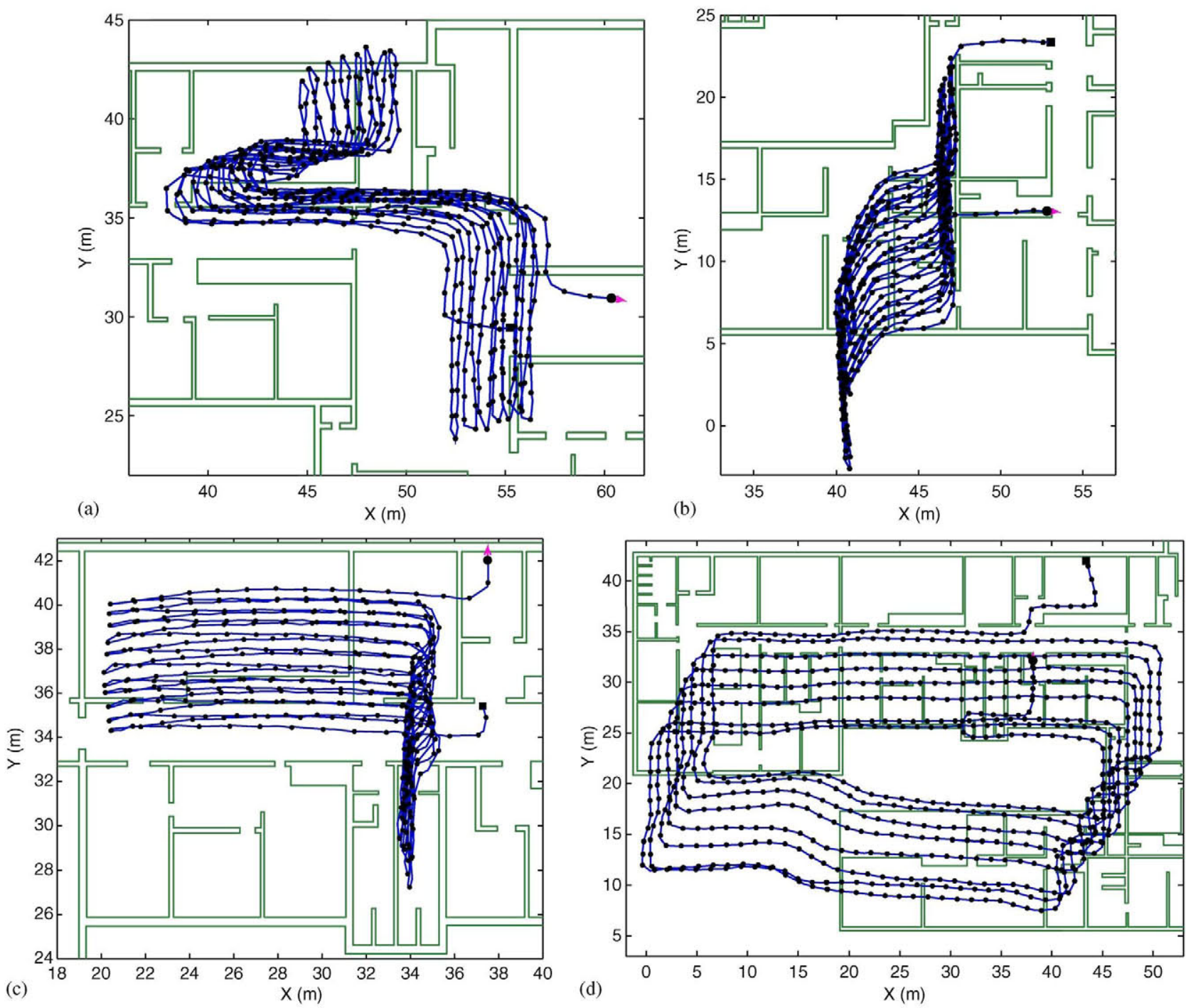

Fig. 8. Different localization tests using the IMU-alone (no RFID information). Several closed cycles are performed along each path (8, 13, 11, and 8 repetitions), with a total length of $600,550,520$, and $1000 \mathrm{~m}$, for tests (a), (b), (c), and (d), respectively. The total start to stop accumulated errors are 5, 10, 7.7, and 11.5 m. In terms of the percentage of the total traveled distance, the errors were $0.8 \%, 1.8 \%, 1.4 \%$, and $1.1 \%$, respectively.

In this section we present the results of the full integrated processing method as presented in Section III. This methodology should limit the total error growth and keep it bounded by a value that depends on the maximum accuracy obtainable by a typical RFID-LPS system (about $2 \mathrm{~m}$ according to most papers in literature [17], [33]). The same trajectories as in Fig. 8 are now displayed in Fig. 9 with the full processing. The position of the installed RFID tags are now displayed on the building map using a red circle.

A first look at Fig. 9 shows that the positioning drift is now eliminated when the RFID information is used. The contribution of RFID ranging information, in spite of having a highly stochastic behavior, is incorporated into the final estimated trajectory as smoothly as in the IMU-alone case (Fig. 8). The total error is of 0.8, 2.3, 1.2, and $1 \mathrm{~m}$, respectively, for Fig. 9(a)-(d). On average, for these 4 tests, the total final error is $1.35 \mathrm{~m}$ (as expected, bounded by the typical accuracy of RFID-based LPS, about $2 \mathrm{~m}$ ). The error percentage with respect to the TTD has no sense in this case since it will tend to zero as the path length increases.
In summary, the integrated IMU+RFID method provides a drift-free solution, with a maximum absolute positioning error dictated by the RFID positioning accuracy, and a very smooth and precise trajectory estimation for short distances, giving details with decimeter resolution for small displacements and turns. Hence, the objective of getting together the best properties of both estimation methods has been achieved.

These localization results are totally satisfactory for pedestrian indoor navigation; however, we also would like to analyze how the performance is influenced by different settings in the selected RSS-to-distance model parameters; and how the RFID tag density in the building influences the positioning results. These topics are treated in the next two subsections.

\section{Sensitivity to RSS Model Parameters}

As presented above, we use a simple and general purpose RSS-to-distance model, whose parameters were found by fitting some test data to actual ranges from RFID reader to tags. For a reference distance $d_{0}$ of $1 \mathrm{~m}$ we found with a high level of 

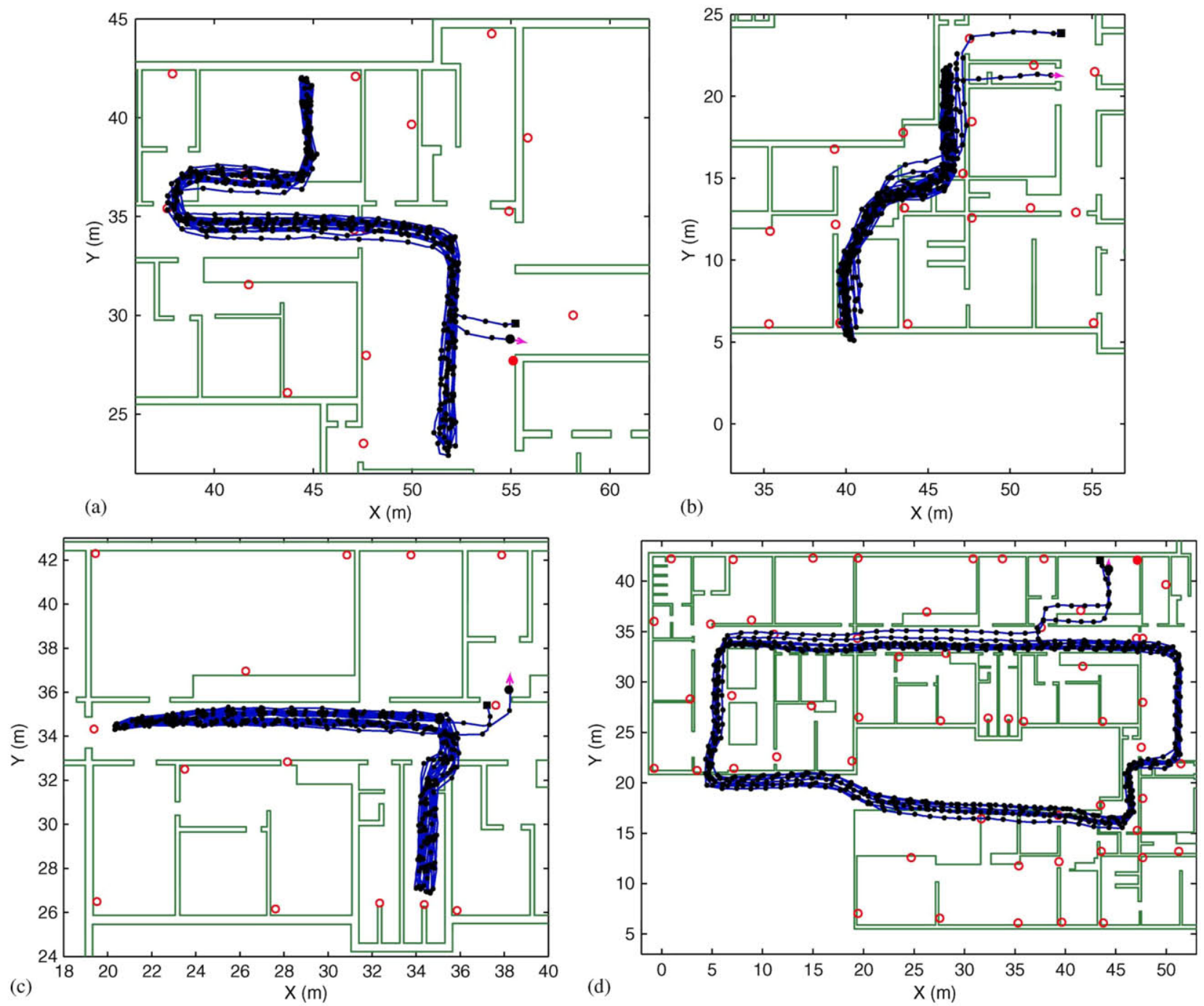

Fig. 9. Position estimation using the proposed integration of RFID to aid the IMU PDR method. The tests are the same of Fig. 8. The error accumulation, as compared to Fig. 8, is strongly attenuated and the start/stop total error is significantly lower: $0.8,2.3,1.2$, and $1.0 \mathrm{~m}$, for tests (a), (b), (c), and (d), respectively. In terms of a percentage of the total travelled distance, the error were $0.15 \%, 0.45 \%, 0.25 \%$, and $0.1 \%$, respectively (Obviously, in the INS + RFID case, it tends to zero as path length increases).

confidence that the $\mathrm{RSS}_{0}$ value (RSS reference value at $1 \mathrm{~m}$ ) is approximately 60 . The other parameter obtained from the fitting was the path loss exponent $p$. We have used until now a value for $p$ of 2.3 , but this value should depend on the building structure (multipath and fading due to walls, doors, furniture), and also could depend on the number of persons and activity in the building (blockage by the trunk of the person to locate, other persons walking around, and so on).

Our hypothesis is that our generic model should be good and behave as an all-terrain model capable of performing satisfactorily for most real indoor scenarios, without requiring an intensive calibration or initial data recording. To test this hypothesis we executed again the positioning algorithms for the different tests presented in Fig. 9, but changing the path loss exponent value $p$. Apart from the default value (2.3) we tested these other ones: $1.5,1.75,2.0,2.6,3.0$, and 4.0. The particular positioning error found for each test and for each specific path loss value is shown in Table II.

It is important to highlight that the average positioning error for the different tests ranges between 1.32 and 2.29, in a path
TABLE II

EFfect of Changing the Path Loss Exponent $(p)$ IN THE RSS-TO-DISTANCE MODEl FOR FOUR DIFFERENT TESTS

\begin{tabular}{|l|l|l|l|l|l|}
\hline \multirow{2}{*}{$\begin{array}{l}\mathrm{p} \text { in model } \\
\left(\mathrm{rss}_{0}=60\right)\end{array}$} & \multicolumn{5}{|c|}{ Positioning Error $(\mathrm{m})$} \\
\cline { 2 - 6 } & test1 & test2 & test3 & test4 & mean \\
\hline 1.5 & 0.8 & 3.66 & 1.54 & 1.45 & $\mathbf{1 . 8 6}$ \\
1.75 & 0.69 & 3.33 & 1.30 & 1.09 & $\mathbf{1 . 6 0}$ \\
2.0 & 0.29 & 2.87 & 1.25 & 0.87 & $\mathbf{1 . 3 2}$ \\
2.3 (Fig.9) & 0.8 & 2.3 & 1.2 & 1.0 & $\mathbf{1 . 3 5}$ \\
2.6 & 1.28 & 1.74 & 1.24 & 1.49 & $\mathbf{1 . 4 3}$ \\
3.0 & 2.00 & 1.16 & 1.34 & 1.8 & $\mathbf{1 . 5 7}$ \\
4.0 & 3.09 & 1.10 & 1.68 & 3.3 & $\mathbf{2 . 2 9}$ \\
\hline
\end{tabular}

loss range from 1.5 to 4.0 , which is a demonstration of the low sensitivity of positing accuracy on the specific selection of the path loss exponent. In fact, as can be better visualized in Fig. 10, the optimum value for $p$ lays in a smooth valley ranging from 1.8 to 2.8 that would account for positioning errors below $1.5 \mathrm{~m}$.

\section{Dependence on Density of RFID Tags}

The results presented until now for the integrated INS + RFID solution use a total of 71 RFID tags distributed quite uniformly 


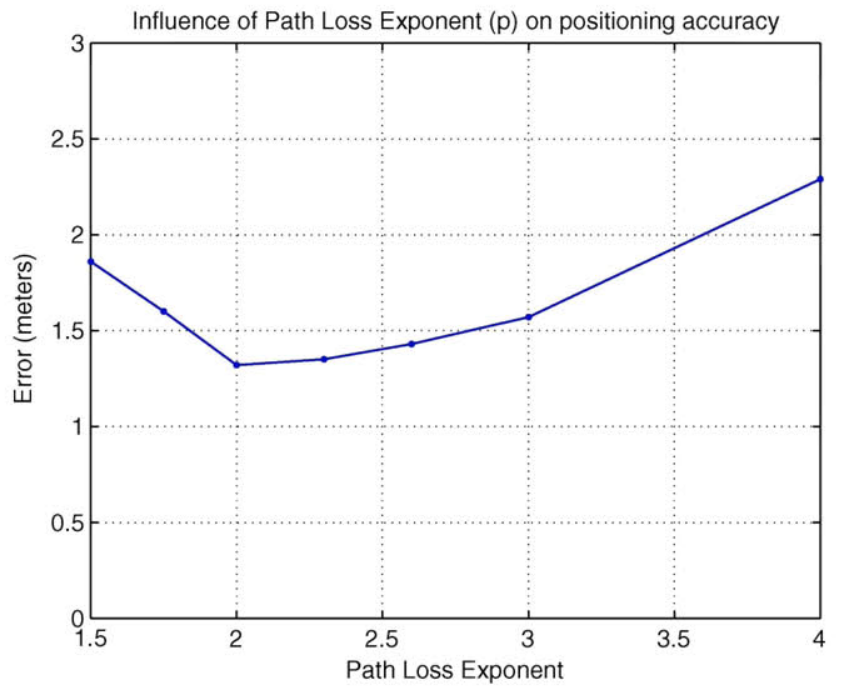

Fig. 10. Path loss exponent influence on positioning accuracy (averaged for four tests of Fig. 9) using all 71 tags.

TABLE III

EFFECT OF CHANGING THE NUMBER OF RFID TAGS FOR FOUR DIFFERENT TESTS

\begin{tabular}{|l|l|l|l|l|l|}
\hline \multirow{2}{*}{$\begin{array}{l}\text { Number } \\
\text { of tags }\end{array}$} & \multicolumn{5}{|c|}{ Positioning Error (m) } \\
\cline { 2 - 6 } 71 (Fig.9) & test1 & test2 & test3 & test4 & mean \\
\hline 50 & 0.8 & 2.3 & 1.2 & 1.0 & $\mathbf{1 . 3 5}$ \\
30 & 1.1 & 5.7 & 1.3 & 1.1 & $\mathbf{1 . 7 2}$ \\
20 & 1.0 & 5.2 & 1.4 & 2.0 & $\mathbf{2 . 4 7}$ \\
14 & 1.2 & 5.0 & 1.3 & 2.3 & $\mathbf{2 . 4 7}$ \\
8 & 1.2 & 5.0 & 2.2 & 2.4 & $\mathbf{2 . 4 7}$ \\
5 & 2.1 & 11.5 & 4.6 & 3.8 & $\mathbf{5 . 5}$ \\
0 (Fig.8) & 5 & 10 & 7.7 & 11.5 & $\mathbf{8 . 5 5}$ \\
\hline
\end{tabular}

on our main building, as presented in Fig. 3. With this tag density, the person wearing the RFID reader will receive approximately 20 tags readings per second. All these tags provide location information, although we know that weak RSS values are less informative in terms of range certainty, i.e., a larger range variance is expected for weak RSS or large distances, as the RSS-to-distance model predicts (7). Nevertheless, it is expected that the more information is available the better positioning accuracy should be obtained. However, we do not know which tag density could be sufficient to keep a satisfactory accuracy while reducing significantly the tag density. This section will study this point.

We will process again the tests presented in Fig. 9 for 71 tags, but in this occasion using a lower number of tags: 50 , $30,20,14,8$, and 5. So, in this study the full recorded RSS information will not be taken into account by the algorithms, but only that coming from the particular set of tags under test. As the most informative tags are supposed to be at short ranges from the person (strong RSS values), we will select tags placed at strategic positions, such as doorways, active for the different groups of tags. The positioning error (start-to-stop distance) for each individual test is presented in Table III. We also included the results with no tags as in Fig. 8 for comparison. The right column of this table shows the average for all tests with the same number of tags. Fig. 11 display this average graphically.

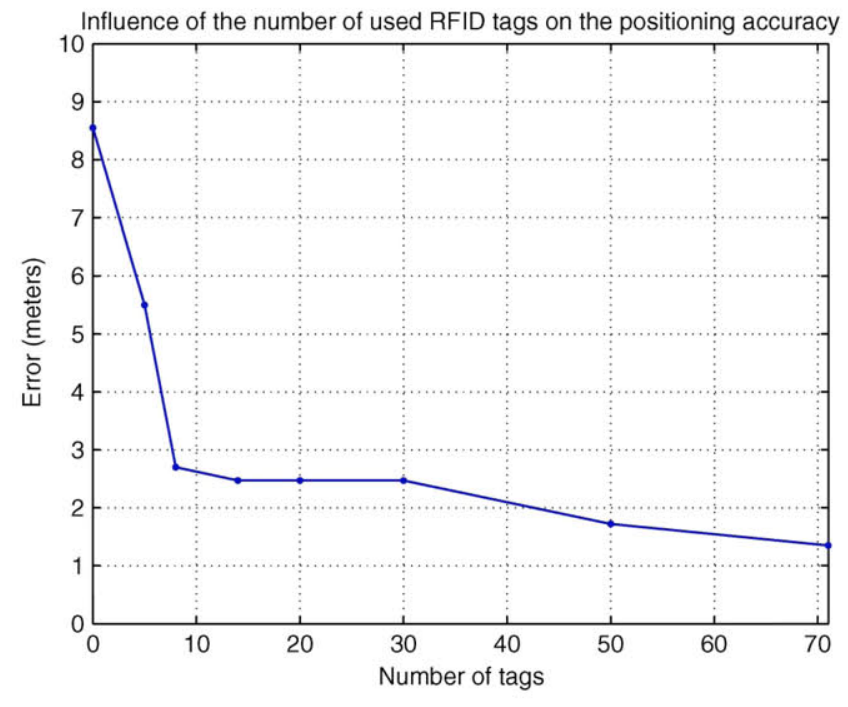

Fig. 11. Influence of the number of RFID tags on the mean positioning accuracy. The case with zero tags corresponds to results in Fig. 8, and the use of 71 was already presented in Fig. 9. A constant path loss exponent $p$ equal to 2.3 is used.

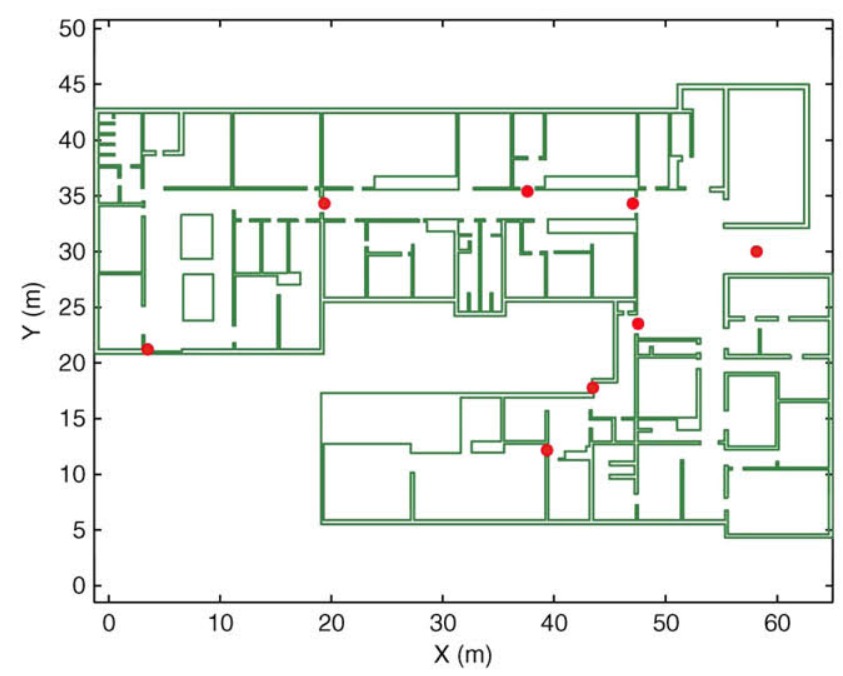

Fig. 12. Distribution of eight RFID tags (red circles) in our main building. This distribution is used in some of the results in Table III and Fig. 11.

It can be seen in Fig. 11 that the addition of just a few tags (5) helps to reduce the original no-tags test from $8.5 \mathrm{~m}$ to $5.5 \mathrm{~m}$, but that improvement is not satisfactory enough. The addition of a few more tags (8 tags distributed as in Fig. 12) improves the accuracy to $2.7 \mathrm{~m}$, which is a significant gain (68\% error reduction) using an infrastructure that only requires 1 tag per $275 \mathrm{~m}^{2}$ (as opposed to 1 tag per $30 \mathrm{~m}^{2}$ using 71 tags).

When we add more tags, from 8 to 71 , we detect a progressive improvement from $2.7 \mathrm{~m}$ to $1.35 \mathrm{~m}$ accuracy, which follows the expected behavior: the larger the density of tags the better the accuracy. With respect to the no-tags case, a total error reduction of $84 \%$ is obtained using 71 tags, and $68 \%$ using only 8 tags. These results indicate that a decent accuracy ( $3 \mathrm{~m})$ can be obtained with few tags; if needed, accuracy can be improved by just adding more tags to approach $1 \mathrm{~m}$ accuracy. 


\section{CONCLUSION}

In this paper, we have presented a tight KF-based INS/RFID integration method for indoor pedestrian localization and navigation. This method uses the residuals between the INS-predicted reader-to-tag ranges and the ranges derived from a generic RSS path-loss model. We consider this RSS-todistance model as a general-purpose one, i.e., it is not too sensitive to the selected path loss exponent in a range from 1.8 to 2.8 . This model was created from an off-line RFID data collection stage (recommended to get insight into the location-dependent RSS fading of a particular building), but not strictly necessary, since the model is expected to work in most brick-made indoor environments. The user gait is not important since the method uses inertial navigation of the foot, and the stride length or way of displacement (fast-slow, lateral, backwards motion) does not affect the INS estimation, consequently an off-line calibration of the user gait is not needed. Pedestrian navigation using only an IMU on the foot, has been shown to slowly drift over long paths or repeated local trajectories. However, our combined integration of INS and RFID information has proven to limit that positioning drift according to the accuracy provided by the network of RFID tags ( $1 \mathrm{~m}$ for high density of tags, and $3 \mathrm{~m}$ for a low density).

The results presented in this paper imply that a very accurate pedestrian navigation or guidance is feasible with IMU and RFID technology. This integrated local positioning technology, which only requires some RFID tags distributed in a building and a person carrying a small computing device (PDA, smartphone) connected to an IMU and RFID reader, can effectively be applied to guide a person from one destination to another in large unfamiliar indoor environments such as airports, office buildings, shopping centers, hospitals, and so on, something specially valuable for the elderly and visually-impaired people. It also could be used in first responder activities to track and control the position of fire-fighters inside buildings; in this case, some RFID tags could be stuck at key positions (e.g., doorways) by the first fire-fighter entering in the building; acting these onthe-fly-placed RFID tags as references for the next incoming personnel. As future work we would like to explore how the use of a map of the building can improve current results, the objective would be to reach $1 \mathrm{~m}$ accuracy with a very low density of tags, or even none at all. We also plan to perform activity recognition based on IMU signals to detect when a person is going up- or down-stairs, on a ramp, opening a door, in an elevator, on a moving escalator or conveyor belt; to obtain position references that can be used to correct the accumulated location error.

\section{REFERENCES}

[1] J. Hightower and G. Borriello, "Location systems for ubiquitous computing," Computer, vol. 34, no. 8, pp. 57-66, Aug. 2001.

[2] A. Jiménez, F. Seco, C. Prieto, and J. Roa, "Tecnologias sensoriales de localización para entornos inteligentes," in Proc. Simposio UCAmI (Granada), España, 2005, pp. 75-86.

[3] Q. Ladetto and B. Merminod, "In step with INS navigation for the blind, tracking emergency crews," GPS World, vol. 13 , no. 10, pp. 30-38, Oct. 2002

[4] L. Fang, P. Antsaklis, L. Montestruque, M. McMickell, M. Lemmon, Y. Sun, H. Fang, I. Koutroulis, M. Haenggi, M. Xie, and X. Xie, "Design of a wireless assisted pedestrian dead reckoning system-The NavMote experience," IEEE Trans. Instrum. Meas., vol. 54, no. 6, pp. 2342-2358, Dec. 2005.

[5] J. Collin, "Investigations of self-contained sensors for personal navigation," Ph.D. thesis, Tampere Univ. Technol., Tampere, Finland, 2006.

[6] R. Levi, "Dead reckoning navigational system using accelerometer to measure foot impacts," U.S. Patent 5583776 , Dec. 10, 1996.

[7] R. Stirling, "Development of a pedestrian navigation system using shoe mounted sensors," M.S. thesis, Northwestern Univ., Evanston, IL, 2005.

[8] Q. Ladetto, J. V. Seeters, S. Sokolowski, Z. Sagan, and B. Merminod, "Digital magnetic compass and gyroscope for dismounted soldier position and navigation," Proc. Sens. Electron. Technol. Panel, NATO Res. Technol. Agency Sens., pp. 1-15, 2002.

[9] A. Jiménez, F. Seco, J. Prieto, and J. Guevara, "A comparison of pedestrian dead-reckoning algorithms using a low-cost MEMS IMU," in Proc. IEEE Int. Symp. Intell. Signal Process., Aug. 2009, pp. 37-42.

[10] A. Chatfield, Fundamentals of High Accuracy Inertial Navigation. Reston, VA: AIAA, 1997.

[11] L. Hutchings, "System and method for measuring movement objects," U.S. Patent 5724 265, Mar. 3, 1998.

[12] E. Foxlin, "Pedestrian tracking with shoe-mounted inertial sensors," IEEE Comput. Graph. Appl., vol. 25, no. 6, pp. 38-46, Nov.-Dec. 2005.

[13] R. Feliz, E. Zalama, and J. García-Bermejo, "Pedestrian tracking using inertial sensors," J. Phys. Agents, vol. 3, no. 1, pp. 35-43, Jan. 2009.

[14] A. Jiménez, F. Seco, J. Prieto, and J. Guevara, "Indoor pedestrian navigation using an INS/EKF framework for Yaw drift reduction and a footmounted IMU," in Proc. 7th WPNC, 2010, vol. 10, pp. 135-143.

[15] N. E1-Sheimy and X. Niu, "The promise of MEMS to the navigation community," in Proc. Inside GNSS, 2007, pp. 46-56.

[16] S. Nassar, "Improving the Inertial Navigation System (INS) error model for INS and INS/DGPS applications," Ph.D. dissertation, Dept. Geomatics Eng., Univ. Calgary, Calgary, AB, Canada, 2003.

[17] A. Koutsou, F. Seco, A. Jiménez, J. Roa, J. Ealo, J. Prieto, and J. Guevara, "Preliminary localization results with an RFID based indoor guiding system," in Proc. IEEE Int. Symp. Intell. Signal Process., Alcalá de Henares, Spain, 2007, pp. 917-922.

[18] V. Amendolare, D. Cyganski, R. Duckworth, S. Makarov, J. Coyne, H. Daempfling, and B. Woodacre, "WPI precision personnel locator system: Inertial navigation supplementation," in Proc. Position, Location, Navig. Symp. IEEE/ION, 2008, pp. 350-357.

[19] I. Skog and P. Handel, "In-car positioning and navigation technologiesa survey," IEEE Trans. Intell. Transp. Syst., vol. 10, no. 1, pp. 4-21, Mar. 2009.

[20] O. Woodman and R. Harle, "RF-based initialisation for inertial pedestrian tracking," in Proc. 7th Int. Conf. Pervasive Comput., Nara, Japan, May 11-14, 2009, p. 238.

[21] H. Wang, H. Lenz, A. Szabo, J. Bamberger, and U. D. Hanebeck, "WLAN-based pedestrian tracking using particle filters and low-cost MEMS sensors," in Proc. 4th Workshop Positioning, Navig. Commun., Mar. 2007, pp. 1-7.

[22] J. Liu, R. Chen, L. Pei, W. Chen, T. Tenhunen, H. Kuusniemi, T. Kroger, and Y. Chen, "Accelerometer assisted robust wireless signal positioning based on a hidden Markov model," in Proc. PLANS IEEE/ION, 2010, pp. 488-497.

[23] V. Renaudin, O. Yalak, P. Tomé, and B. Merminod, "Indoor navigation of emergency agents," Eur. J. Navig., vol. 5, no. 3, pp. 36-45, Jul. 2007.

[24] K. Zhang, M. Zhu, G. Retscher, F. Wu, and W. Cartwright, "Threedimension indoor positioning algorithms using an integrated RFID/INS system in multi-storey buildings," in Proc. Location Based Services Tele Cartography II, 2009, pp. 373-386.

[25] M. Zhu, K. Zhang, W. Cartwright, and G. Retscher, "Possibility studies of integrated INS/RFID positioning methods for personal positioning applications," in Proc. Global Navig. Satellite, 2009, pp. 1-9.

[26] G. Retscher and Q. Fu, "Integration of RFID, GNSS and DR for ubiquitous positioning in pedestrian navigation," J. Global Positioning Syst. vol. 6, no. 1, pp. 56-64, 2007.

[27] G. Retscher and Q. Fu, "Active RFID trilateration for indoor positioning," Coordinates, pp. 10-15, May 2008.

[28] S. Mazuelas, A. Bahillo, R. M. Lorenzo, P. Fernandez, F. A. Lago, E. Garcia, J. Blas, and E. J. Abril, "Robust indoor positioning provided by real-time RSSI values in unmodified WLAN networks," IEEE J. Sel. Topics Signal Process., vol. 3, no. 5, pp. 821-831, Oct. 2009.

[29] G. Retscher and Q. Fu, "Continuous indoor navigation with RFID and INS," in Proc. Position Location Navig., 2010, pp. 102-112.

[30] Q. Fu and G. Retscher, "Using RFID and INS for indoor positioning," in Proc. Location Based Services TeleCartography II, 2009, pp. 421-438.

[31] J. A. Farrell and M. Barth, The Global Positioning System and Inertial Navigation. New York: McGraw-Hill, 1999. 
[32] S. Seidel and T. Rappaport, "914 MHz path loss prediction models for indoor wireless communications in multifloored buildings," IEEE Trans. Antennas Propag., vol. 40, no. 2, pp. 207-217, Feb. 1992.

[33] F. Seco, C. Plagemann, A. Jiménez, and W. Burgard, "Improving RFIDbased indoor positioning accuracy using Gaussian processes," in Proc. Int. Conf. Indoor Positioning Indoor Navig., 2010, pp. 15-17.

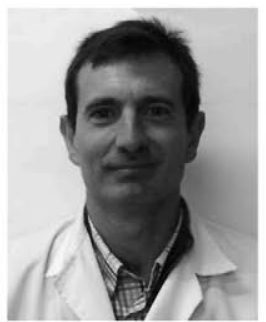

Antonio Ramón Jiménez Ruiz received the degree in physics and computer science and the Ph.D. degree in physics from the Universidad Complutense de Madrid, Madrid, Spain, in 1991 and 1998, respectively.

From 1991 to 1993, he worked in industrial laser applications with the Technological Center of Madrid. Since 1994, he has been a Researcher within the Instituto de Automática Industrial (now named Centro de Automática y Robótica), belonging both to the largest Public-funded Agency for Research in Spain (Consejo Superior de Investigaciones Científicas). Since 2005, he belongs to the LOPSI group, specialized in designing local positioning solutions for indoor localization and navigation. His current research interests include: sensors, signal processing, and algorithms applied to the precise indoor/outdoor localization of persons and robots. He has a special interest in creating prototypes to demonstrate the functionality of local positioning Systems using acoustic/ultrasonic and RFID technology, as well as, Pedestrian Dead-Reckoning solutions using foot-mounted IMUs. He is author and reviewer of many international journals and communications in the field.

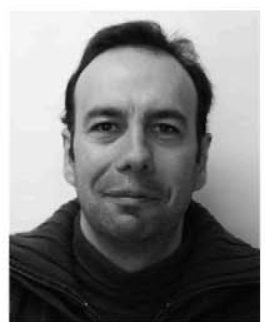

Fernando Seco Granja was born in Madrid, Spain, in 1972. He received the degree in physics from the Universidad Complutense of Madrid, Madrid, Spain, in 1996 and the Ph.D. degree in physics from the Universidad Nacional de Educación a Distancia, Madrid, Spain, in 2002. His dissertation dealt with the generation of ultrasonic waves applied to a magnetostrictive linear position sensor.

Since 1997, he has been with the Centro de Automática y Robótica, Consejo Superior de Investigaciones Científicas-UPM, Madrid, Spain, where he holds a research position. His main research interest lies in the design and development of local positioning systems, particularly those based on ultrasound and RFID, and specifically on the topics of signal processing of ultrasonic signals, multilateration algorithms, and Bayesian localization methods.

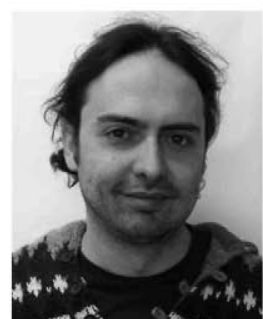

José Carlos Prieto Honorato was born in León, Spain, in 1978. He received the Technical degree in industrial electronics and the B.S. degree in electronics engineering from the Universidad de Extremadura, Badajoz, Spain, in 1999 and 2003, respectively, and the Master's degree in robotics from the Universidad Politécnica de Madrid, Madrid, Spain, in 2007. He is currently working toward the Doctoral degree in robotics with the Universidad de Alcalá, Madrid, Spain.

Since 2004, he has been a Researcher with the Centro de Automática y Robótica, Consejo Superior de Investigaciones Científicas-UPM, Madrid, Spain. His research interests are focused in localization systems, mainly those based in ultrasonic signals, with special emphasis in signal design and processing, positioning algorithms, robustness, standardization, optimal configurations, calibration methods, and development of new transducers.

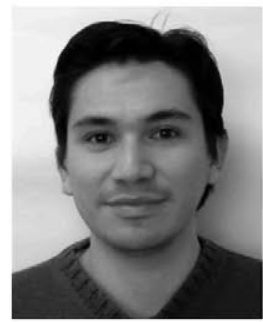

Jorge I. Guevara Rosas was born in Lima, Peru, in 1978 . He received the B.S. degree in electronics engineering from the Universidad Católica Nuestra Señora de la Asunción, Asunción, Paraguay, in 2004. $\mathrm{He}$ is currently working toward the Ph.D. degree in electric engineering with the Centro de Automática y Robótica, Consejo Superior de Investigaciones Científicas-UPM, Madrid, Spain.

His research interests are in the area of localization systems, in particular, automatic calibration methods for ultrasonic positioning systems. 